\title{
INTERPOLATION GEVREY DANS LES DOMAINES DE TYPE FINI DE $\mathbb{C}^{2}$
}

\author{
VINCENT THILLIEZ \\ Mathématiques, CNRS-URA D751 \\ Université des Sciences et Technologies de Lille, F-59655 Villeneuve d'Ascq Cedex, France
}

0. Introduction. La notion d'interpolation à l'ordre infini pour les fonctions holomorphes désigne un lointain dérivé du problème de Borel et Carleman concernant la construction d'une fonction $C^{\infty}$ sur la droite, à dérivées prescrites en un point. Il s'agit ici, étant donnés un point $p$ (plus généralement un compact $E) \mathrm{du}$ bord d'un domaine $\Omega$ de $\mathbb{C}^{n}$ et une famille $F=\left(F_{J}\right)_{J \in \mathbb{N}^{2 n}}$ de nombres complexes (resp. d'applications continues sur $E$, à valeurs complexes), de préciser sous quelles conditions on peut trouver une fonction $f$ holomorphe sur $\Omega$ dont toutes les dérivées $D^{J} f\left(J \in \mathbb{N}^{2 n}\right)$ admettent $F_{J}$ pour valeur au bord en $p$ (resp. sur $E$ ). Bien sûr, outre une condition nécessaire triviale de $\bar{\partial}$-platitude pour $F$, la nature du problème dépend essentiellement de deux aspects :

(i) la géométrie de $\partial \Omega$,

(ii) la régularité exigée sur $f$ et, par conséquent, sur $F$ (conditions de croissance par exemple).

On connaît en particulier, dans le cas où $\Omega$ est à bord de classe $C^{2}$ strictement pseudoconvexe dans $\mathbb{C}^{n}$, une solution du problème dans les classes de fonctions suivantes :

(i) $A^{\infty}(\Omega):=H(\Omega) \cap C^{\infty}(\bar{\Omega})$ (voir par ex. [CC1]), où on a noté $H(\Omega)$ l'espace des fonctions holomorphes dans $\Omega$,

(ii) $H G^{1+\alpha}(\Omega):=H(\Omega) \cap G^{1+\alpha}(\Omega)$ pour $\alpha>1$ (voir $[\mathrm{CC} 2]$ ), où $G^{1+\alpha}(\Omega)$ désigne la classe de Gevrey standard d'indice $1+\alpha$ sur $\Omega$.

On rappelle qu'une fonction $f$ de $C^{\infty}(\bar{\Omega})$ appartient à $G^{1+\alpha}(\Omega)\left(\alpha \in \mathbb{R}_{+}\right)$s'il existe deux constantes $M$ et $K, M>0, K \geq 1$ (dépendant de $f$ ), telles que l'on

1991 Mathematics Subject Classification: 32A40, 26E10.

The paper is in final form and no version of it will be published elsewhere. 
ait, pour tout $J$ de $\mathbb{N}^{2 n}$ et tout $z$ de $\Omega$,

$$
\left|D^{J} f(z)\right| \leq M K^{j} j !^{1+\alpha},
$$

où $j$ est la longueur de $J$. (Il s'agit bien ici de régularité jusqu'au bord.)

Si l'on regarde les études respectives de (i) et (ii) dans [CC1], [CC2], on constate que dans le cas Gevrey, la nécessité d'avoir à considérer des conditions de croissance sur les dérivées impose une analyse plus délicate, distinguant les directions complexes tangentielles à $\partial \Omega$ de la direction transverse. Ceci est lié au phénomène (initialement mis en évidence par Stein dans le contexte Lipschitz [St][GrSt]) qui veut qu'en règle générale, une fonction holomorphe sur un ouvert à bord lisse satisfaisant une régularité jusqu'au bord donnée, possède en fait une régularité au moins double (selon la géométrie du domaine) dans les directions complexes tangentielles.

Ces considérations apparaissent aussi clairement dans un travail de Bruna et Ortega [BO] sur l'interpolation (à l'ordre fini) pour les classes de Hölder de la boule.

Partant de ces remarques, nous avons étendu dans [T1][T2] les résultats de [CC2] au cas d'un voisinage d'un point $z^{0}$ de type fini sur le bord d'un domaine pseudoconvexe à bord lisse $\Omega$ dans $\mathbb{C}^{2}$. Nous résumons ici les résultats obtenus.

Il est d'abord nécessaire de définir certains outils géométriques précis.

1. Outils géométriques. Soient $\Omega$ un domaine de $\mathbb{C}^{2}$ à frontière de classe $C^{\infty}, z^{0}$ un point de $\partial \Omega$ et $r$ une fonction définissante pour $\Omega$ au voisinage de $z^{0}$. Pour $z=\left(z_{1}, z_{2}\right)$ dans $\mathbb{C}^{2}$, on note $x_{j}=\operatorname{Re} z_{j}$ et $y_{j}=\operatorname{Im} z_{j}(j=1,2)$. On peut supposer $\frac{\partial r}{\partial x_{2}}\left(z^{0}\right)>0$; il est alors démontré dans [Ca] qu'il existe dans un voisinage convenable $U$ de $z^{0}$, pour tout entier $m$, des applications $d_{0}, d_{1}, \ldots, d_{m}$ de classe $C^{\infty}$ sur $U$, uniques telles que, pour tout $z^{\prime}$ de $U$, l'application $\Phi_{z^{\prime}}$ qui à $\zeta$ de $\mathbb{C}^{2}$ associe $z$ donné par :

$$
z_{1}=z_{1}^{\prime}+\zeta_{1}, \quad z_{2}=z_{2}^{\prime}+d_{0}\left(z^{\prime}\right) \zeta_{2}+\sum_{j=1}^{m} d_{j}\left(z^{\prime}\right) \zeta_{1}^{j},
$$

définisse un changement de coordonnées holomorphes dans $\mathbb{C}^{2}$ et telles que la fonction $\rho_{z^{\prime}}=\operatorname{ro} \Phi_{z^{\prime}}$, définissante pour $\Phi_{z^{\prime}}^{-1}(\Omega)$ au voisinage de 0 , admette un développement de la forme

$$
\rho_{z^{\prime}}(\zeta)=r\left(z^{\prime}\right)+\operatorname{Re} \zeta_{2}+\sum_{\substack{j+k \leq m \\ j \geq 1, \bar{k} \geq 1}} a_{j k}\left(z^{\prime}\right) \zeta_{1}^{j} \bar{\zeta}_{1}^{k}+\mathcal{O}\left(\left|\zeta_{1}\right|^{m+1}+|\zeta|\left|\zeta_{2}\right|\right) .
$$

On pose, pour $2 \leq \ell \leq m, A_{\ell}\left(z^{\prime}\right)=\max _{j+k=\ell}\left|a_{j k}\left(z^{\prime}\right)\right|$. On fait alors l'hypothèse que le point $z^{0}$ est de type fini $m$, ce qui signifie que l'on a $A_{\ell}\left(z^{0}\right)=0$ pour $\ell<m$ et $A_{m}\left(z^{0}\right) \neq 0$. Quitte à rétrécir $U$, on a alors $A_{m} \neq 0$ dans $U$ et on peut définir, pour $z^{\prime}$ dans $U$ et $\delta$ réel strictement positif, les quantités

$$
\theta\left(z^{\prime}\right)=\min \left\{\ell ; A_{\ell}\left(z^{\prime}\right) \neq 0\right\}
$$


(en particulier, pour $z^{\prime} \in U \cap \partial \Omega, \theta\left(z^{\prime}\right)$ est le type de $z^{\prime}$ ),

$$
\tau\left(z^{\prime}, \delta\right)=\left(\sum_{\ell=2}^{m}\left(\frac{A_{\ell}\left(z^{\prime}\right)}{\delta}\right)^{1 / \ell}\right)^{-1}
$$

et

$$
T\left(z^{\prime}, \delta\right)=\frac{\log \delta}{\log \left(\tau\left(z^{\prime}, \delta\right) / \tau\left(z^{\prime}, 1\right)\right)} .
$$

La quantité précédente est un analogue continu du $T$ de Catlin [Ca], avec une normalisation convenable qui permet, par des considérations élémentaires de fonctions convexes, de montrer que $T\left(z^{\prime}, \delta\right)$ est une fonction croissante de $\delta$, comprise entre $\theta\left(z^{\prime}\right)$ et $m$, et se prolonge en 0 en posant $T\left(z^{\prime}, 0\right)=\theta\left(z^{\prime}\right)$.

On définit enfin une famille de pseudoboules ("bent polydisks" de [Ca]) par

$$
Q_{\delta}\left(z^{\prime}\right)=\Phi_{z^{\prime}}\left(R_{\delta}\left(z^{\prime}\right)\right)
$$

où $R_{\delta}\left(z^{\prime}\right)$ est le bidisque ouvert de centre 0 , de birayon $\left(\tau\left(z^{\prime}, \delta\right), \delta\right)$. On leur associe une pseudodistance [NSW] définie, pour $z^{\prime}$ et $z$ dans $U$, par

$$
\delta\left(z^{\prime}, z\right)=\inf \left\{\eta>0 ; z \in Q_{\eta}\left(z^{\prime}\right)\right\} .
$$

La quantité $\Theta\left(z^{\prime}, z\right)=T\left(z^{\prime}, \delta\left(z^{\prime}, z\right)\right)$ décrit alors la taille de la plus petite pseudoboule de centre $z^{\prime}$ contenant $z$ en ce sens que, de façon simpliste, cette taille est de l'ordre de $\delta^{1 / \Theta}$ dans la direction complexe-tangentielle et $\delta$ dans la direction transverse. On a une liste de propriétés résumée ci-dessous.

\subsection{Proposition. On a}

(i) $2 \leq \theta\left(z^{\prime}\right) \leq \Theta\left(z^{\prime}, z\right) \leq m$,

(ii) $\delta\left(z^{\prime}, z\right) \leq \delta\left(z^{\prime}, z^{\prime \prime}\right)$ entraîne $\Theta\left(z^{\prime}, z\right) \leq \Theta\left(z^{\prime}, z^{\prime \prime}\right)$,

(iii) $\Theta\left(z^{\prime}, z^{\prime}\right)=\theta\left(z^{\prime}\right)$,

(iv) $\frac{1}{C}\left(\left|\zeta_{1}\right|^{\Theta\left(z^{\prime}, z\right)}+\left|\zeta_{2}\right|\right) \leq \delta\left(z^{\prime}, z\right) \leq C\left(\left|\zeta_{1}\right|^{\Theta\left(z^{\prime}, z\right)}+\left|\zeta_{2}\right|\right)$ pour $z=\Phi_{z^{\prime}}(\zeta)$,

(v) $\left|\Theta\left(z^{\prime}, z\right)-\Theta\left(z, z^{\prime}\right)\right| \leq C /\left|\log \delta\left(z^{\prime}, z\right)\right|$,

où $C$ est une constante, $C \geq 1$, ne dépendant que de la géométrie de $\Omega$.

On construit ensuite des régions d'approche. Pour $a$ et $t$ réels avec $0<a \leq 1$, $0<t \leq 1$ et pour $z^{\prime}$ dans $U \cap \partial \Omega$, on désigne par $P^{t, a}\left(z^{\prime}\right)$ le bidisque fermé de centre $\left(0,-t h / d_{0}\left(z^{\prime}\right)\right)$ et de birayon $\left(\tau\left(z^{\prime}, a\left|r\left(z_{1}^{\prime}, z_{2}^{\prime}-t h\right)\right|\right), a\left|r\left(z_{1}^{\prime}, z_{2}^{\prime}-t h\right)\right|\right)$, où $h$ est une constante positive assez petite. On pose enfin, pour $0<t_{0} \leq 1$ et $0<a_{0} \leq 1$

$$
U_{z^{\prime}}^{t_{0}, a_{0}}=\Phi_{z^{\prime}}\left(\bigcup_{0 \leq t \leq t_{0}} P^{t, a_{0}}\left(z^{\prime}\right)\right) .
$$

Il est à noter que la construction classique [NSW] de régions d'approches équivalentes à celles-ci consiste d'abord à définir des pseudoboules $B_{\delta}\left(z^{\prime}\right)$ vivant sur $\partial \Omega$, puis à définir $U_{z^{\prime}}^{t_{0}, a_{0}}$ comme ensemble des points $z$ avec $|r(z)|<t_{0}$, qui se projettent dans $B_{a_{0}|r(z)|}\left(z^{\prime}\right)$. La méthode d'empilement de bidisques de Catlin 
décrite auparavant fournit une version élémentaire, quoique moins intrinsèque, de cette construction.

Une des caractéristiques essentielles des régions d'approche réside dans la propriété suivante.

1.2. Proposition. Il existe une constante $a, 0<a \leq 1$, ne dépendant que de la géométrie de $\Omega$, telle que, pour $0<a_{0} \leq a, 0<t_{0} \leq 1$ et pour tous points $z^{\prime}$ de $U \cap \partial \Omega$ et $z$ de $U_{z^{\prime}}^{t_{0}, a_{0}}$, on ait

$$
\frac{1}{C}|r(z)| \leq \delta\left(z^{\prime}, z\right) \leq C|r(z)|
$$

où $C$ est une constante, $C \geq 1$, ne dépendant que de la géométrie de $\Omega$.

On sera amené à utiliser, dans les estimations Gevrey que l'on donnera, des itérés de champs de vecteurs au lieu des dérivations naturelles. Cependant, si on considère les champs $L_{1}$ et $L_{2}$ respectivement complexe-tangentiel et transverse définis par

$$
L_{1}=\frac{\partial}{\partial z_{1}}-\left(\frac{\partial r}{\partial z_{2}}\right)^{-1} \frac{\partial r}{\partial z_{1}} \frac{\partial}{\partial z_{2}} \quad \text { et } \quad L_{2}=\frac{\partial}{\partial z_{2}},
$$

on se heurte à deux obstructions :

(i) la régularité de $r$ n'étant a priori que $C^{\infty}$, des itérés de longueur arbitrairement grande de $L_{1}$ (nécessaire pour des estimations Gevrey) risquent de comporter des termes parasites qui "explosent",

(ii) $L_{1}$ et $L_{2}$ ne commutent généralement pas.

Il est plus naturel d'adopter les champs de vecteurs $X_{z^{\prime}, j}, j=1,2$, associés aux coordonnées de Catlin :

$$
X_{z^{\prime}, j}=\left(\Phi_{z^{\prime}}\right)_{*}\left(\partial / \partial \zeta_{j}\right)
$$

On peut vérifier qu'à l' "origine" $z^{\prime}$, on a $X_{z^{\prime}, 1}\left(z^{\prime}\right)=L_{1}\left(z^{\prime}\right)$. On a en outre $X_{z^{\prime}, 2}=d_{0}\left(z^{\prime}\right) L_{2}$.

Enfin, soit $P=\left(p^{\prime}, p^{\prime \prime}\right)$ un biindice. On note $p=|P|=p^{\prime}+p^{\prime \prime}$. La notation $X_{z^{\prime}, j}^{P}\left(\operatorname{resp} . L_{j}^{P}, \zeta_{j}^{P}\right)$ désigne $X_{z^{\prime}, j}^{p^{\prime}} \bar{X}_{z^{\prime}, j}^{p^{\prime \prime}}\left(\right.$ resp. $\left.L_{j}^{p^{\prime}} \bar{L}_{j}^{p^{\prime \prime}}, \zeta_{j}^{p^{\prime}} \bar{\zeta}_{j}^{p^{\prime \prime}}\right)$. Si $Q$ est un second biindice, $\left.X^{P Q}, \zeta^{P Q}\right)$ désigne $X_{z^{\prime}, 1}^{P} X_{z^{\prime}, 2}^{Q}\left(\operatorname{resp} . L_{1}^{P} L_{2}^{Q}, \zeta_{1}^{P} \zeta_{2}^{Q}\right)$.

\section{Classes de Gevrey non isotropes}

2.1.DÉfinition. Soit $\alpha>0$. Une fonction $f$ dans la classe de Gevrey standard $G^{1+\alpha}(\Omega \cap U)$ (cf. introd.) est dite appartenir à la classe de Gevrey non isotrope $G_{N I}^{1+\alpha}(\Omega \cap U)$ s'il existe des constantes $M, K, t_{f}, a_{f}$ avec $M \geq 0, K \geq 1,0<t_{f} \leq 1$, $0<a_{f} \leq a$, dépendant de $f$, telles que pour tous $z^{\prime}$ de $U \cap \partial \Omega$, $z$ de $U_{z^{\prime}}^{t_{f}, a_{f}}$, tous biindices $P$ et $Q$ et tout réel $\lambda$ satisfaisant

$$
0 \leq \lambda \leq q\left(1-\frac{1}{\Theta\left(z^{\prime}, z\right)}\right),
$$


on ait

(*) $\quad|r(z)|^{\lambda}\left|X_{z^{\prime}}^{P Q} f(z)\right| \leq M K^{\left(p / \Theta\left(z^{\prime}, z\right)\right)+q-\lambda}(p+q) !\left[\frac{p}{\Theta\left(z^{\prime}, z\right)}+q-\lambda\right] !^{\alpha}$.

Soit $H G^{1+\alpha}(\Omega \cap U)$ la classe des fonctions holomorphes appartenant à la classe de Gevrey standard $G^{1+\alpha}(\Omega \cap U)$. Le résultat qui suit a motivé la définition (2.1).

2.2. THÉORÈME. On a l'inclusion

$$
H G^{1+\alpha}(\Omega \cap U) \subset G_{N I}^{1+\alpha}(\Omega \cap U) .
$$

En inspectant $(*)$, on voit que l'on a bien mis en évidence un gain de régularité Gevrey : $1+\alpha / \Theta\left(z^{\prime}, z\right)$ au lieu de $1+\alpha$, dans la direction $X_{z^{\prime}, 1}(z)$, pour $z$ dans $U_{z^{\prime}}^{t_{f}, a_{f}}$. Cette régularité améliorée peut varier continûment (cf. (1.1)) de $1+\alpha / 2$ à $1+\alpha / m$ en fonction du type de $z^{\prime}$ et de la position relative de $z$ et $z^{\prime}$. Pour un domaine strictement pseudoconvexe, on a $m=2$ : on retrouve alors la théorie à gain fixe de [CC2].

2.3. R e m a rqu e. Dans le cas d'un domaine $\Omega$ rigide polynomial, c'est-à-dire lorsque $r(z)=\operatorname{Re} z_{2}+P\left(z_{1}, \bar{z}_{1}\right)$ où $P$ est un polynôme homogène de degré $m$ sans terme harmonique, il est possible, dans la condition $(*)$ de la définition $(2.1)$, de remplacer $X_{z^{\prime}}^{P Q} f$ par $L^{P Q} f$. L'équivalence des deux estimations est liée au fait que pour un tel domaine, les obstructions (i) et (ii) à l'utilisation des $L_{j}$ évoquées précédemment disparaissent.

3. Interpolation. Soit $E$ un sous-ensemble compact de $U \cap \partial \Omega$, dont chaque point est de type $m$. On définira un jet $F$ sur $E$ par la donnée d'une famille $\left(F_{P Q}\right)_{P \in \mathbb{N}^{2}, Q \in \mathbb{N}^{2}}$ d'applications continues sur $E$, à valeurs complexes. Pour tout $z^{\prime}$ de $E$ et tout entier naturel $n$, on définit le polynôme de Taylor non isotrope $T_{n}\left(z^{\prime}, F\right)$ du jet $F$ par

$$
T_{n}\left(z^{\prime}, F\right)(z)=\sum_{\substack{I \in \mathbb{N}^{2}, J \in \mathbb{N}^{2} \\ i / m+j \leq n}} \frac{1}{I ! J !} F_{I J}\left(z^{\prime}\right) \zeta^{I J},
$$

où $z=\Phi_{z^{\prime}}(\zeta)$.

Le jet $F$ sera dit appartenir à la classe non isotrope $G_{N I}^{1+\alpha}(E)(\alpha>0)$ s'il existe deux constantes $M$ et $K, M>0$ et $K \geq 1$ telles que les propriétés suivantes soient vérifiées :

(i) Pour tous biindices $P$ et $Q$ et tout $z^{\prime}$ de $E$, on a

$$
\left|F_{P Q}\left(z^{\prime}\right)\right| \leq M K^{(p / m)+q}(p+q) ![(p / m)+q] !^{\alpha} .
$$

(ii) Pour tous biindices $P$ et $Q$, tout entier naturel $n$, tous points $z$ et $z^{\prime}$ de $E$, et pourvu que l'on ait $p / m+q \leq n$, on a

$\left|F_{P Q}(z)-X_{z}^{P Q} T_{n}\left(z^{\prime}, F\right)(z)\right| \leq M K^{(p / m)+q+1}(p+q) !(n !)^{\alpha} \delta\left(z^{\prime}, z\right)^{n+(1 / m)-((p / m)+q)}$. 
Cette définition est motivée par la remarque suivante : pour toute fonction $f$ de $G_{N I}^{1+\alpha}(\Omega \cap U)$, la famille $\left(F_{P Q}\right)_{P \in \mathbb{N}^{2}, Q \in \mathbb{N}^{2}}$ donnée par

$$
F_{P Q}\left(z^{\prime}\right)=\left(X_{z^{\prime}}^{P Q} f\right)\left(z^{\prime}\right), \quad z^{\prime} \in E,
$$

définit un jet de $G_{N I}^{1+\alpha}(E)$. En effet, la propriété (i) résulte de (*) appliqué avec $z=z^{\prime}, \lambda=0$, puisque l'on a $\Theta\left(z^{\prime}, \cdot\right) \equiv m$ pour $z^{\prime}$ de type $m$. La propriété (ii) provient d'un cas particulier de la formule de Taylor non isotrope démontrée sous une forme générale technique dans [T1].

Le théorème qui suit fournit une réciproque à cette remarque.

3.1. Théorème D'extension À LA Whitney. Soit $F$ un jet de $G_{N I}^{1+\alpha}(E)$. Alors il existe $f$ dans $G_{N I}^{1+\alpha}(\Omega \cap U)$ telle que l'on ait

$$
\left(X_{z^{\prime}}^{P Q} f\right)\left(z^{\prime}\right)=F_{P Q}\left(z^{\prime}\right)
$$

pour tout $z^{\prime}$ de $E$ et tous biindices $P$ et $Q$.

3.2. Hypothèses pour l'interpolation. Dans toute la suite on suppose que $\Omega$ est pseudoconvexe au voisinage de $z^{0}$ (donc $m$ est pair) et que $\alpha$ est un réel fixé avec $\alpha>1$. Cette condition sur $\alpha$ est naturelle car, un ensemble d'interpolation pour $H G^{1+\alpha}(\Omega \cap U)$ étant aussi un ensemble de non unicité, la classe considérée doit être non quasianalytique (ce qui n'est pas le cas pour $\alpha \leq 1$, voir [Ch]). On suppose enfin que $E$ satisfait les hypothèses suivantes :

(i) Chaque point de E est de type strict $m$ (voir [Bl]), c'est-à-dire qu'il existe une constante $c$ strictement positive telle que l'on ait

$$
\sum_{\substack{j+k=m \\ j \geq 1, k \geq 1}} a_{j k}\left(z^{\prime}\right) \zeta_{1}^{j} \bar{\zeta}_{1}^{k} \geq c\left|\zeta_{1}\right|^{m}
$$

pour tous $\zeta_{1}$ de $\mathbb{C}$ et $z^{\prime}$ de $E$ (on sait que $a_{j k}\left(z^{\prime}\right)=0$ pour $j+k<m$ ).

(ii) Pour tout $z^{\prime}$ de $E$ et tout $z=\Phi_{z^{\prime}}(\zeta)$ de $U \cap \bar{\Omega}$, on $a \operatorname{Re} \zeta_{2} \leq 0$.

(iii) Pour toute pseudoboule $Q_{R}$ de rayon $R, 0<R<1$, on a

$$
\int_{0}^{R} N_{\varepsilon}\left(E \cap Q_{R}\right) \frac{d \varepsilon}{\varepsilon^{1 / \alpha}} \leq C R^{1-1 / \alpha},
$$

où $C$ est une constante positive et où $N_{\varepsilon}\left(E \cap Q_{R}\right)$ désigne le nombre minimum de pseudoboules de rayon $\varepsilon$ recouvrant $E \cap Q_{R}$.

3.3. ThÉorème D'interpolation. Sous les hypothèses (3.2) précédentes, quitte à rétrécir $U$, il existe pour tout jet $F$ dans $G_{N I}^{1+\alpha}(E), \bar{\partial}$-plat, une fonction $f$ dans $H G^{1+\alpha}(\Omega \cap U)$ telle que l'on ait

$$
\left(X_{z^{\prime}}^{P Q} f\right)\left(z^{\prime}\right)=F_{P Q}\left(z^{\prime}\right)
$$

pour tous biindices $P$ et $Q$ et tout $z^{\prime}$ de $E$.

La preuve utilise d'abord une extension non holomorphe fournie par (3.1). On obtient ensuite l'holomorphie via un procédé de division dans l'algèbre 
$G_{N I}^{1+\alpha}(\Omega \cap U)$, joint à la résolution du $\bar{\partial}$ dans les classes de Gevrey due à ChaumatChollet [CC3].

3.4. Commentaires. (i) Dans le cas où $E$ est réduit à un point, on peut se dispenser des hypothèses (3.2)(ii) et (iii), et même de l'hypothèse (3.2)(i) pour $\alpha>\alpha_{0}, \alpha_{0}$ assez grand. La valeur optimale de $\alpha_{0}$ est une question ouverte.

(ii) Les hypothèses (3.2)(i) et (ii) ne sont "pas trop restrictives", en ce sens que des exemples très courants de domaines entrent dans le cadre étudié : pseudoellipsoïdes complexes et rééls, domaines rigides polynômiaux de type strict ... L'hypothèse (3.2)(iii) implique, elle, que les ensembles d'interpolation considérés ne peuvent être trop "gros" : plaçons-nous par exemple sur $\Omega=\left\{z ; \operatorname{Re} z_{2}+\left|z_{1}\right|^{4}<\right.$ $0\}$, pour lequel $E$ doit être contenu dans l'axe $\{(0, i t) ; t \in \mathbb{R}\}$. On peut voir facilement qu'ici (iii) impose que la dimension de Hausdorff de $E$ soit au plus $1-1 / \alpha$. En adaptant une construction de [CC4], on peut même donner un exemple où cette dimension est exactement $1-1 / \alpha$.

\section{Références}

[Bl] T. Bloom, $C^{\infty}$ peak functions for pseudoconvex domains of strict type, Duke Math. J. 45 (1978), 133-147.

[BO] J. Bruna and J. M. Ortega, Interpolation by holomorphic functions smooth to the boundary in the unit ball of $\mathbb{C}^{n}$, Math. Ann. 274 (1986), 527-575.

[Ca] D. Catlin, Estimates of invariant metrics on weakly pseudoconvex domains of dimension two, Math. Z. 200 (1989), 429-466.

[CC1] J. Chaumat et A.-M. Chollet, Ensemble de zéros et d'interpolation à la frontière de domaines strictement pseudoconvexes, Ark. Mat. 24 (1985), 27-57.

[CC2] -, - Classes de Gevrey non isotropes et application à l'interpolation, Ann. Scuola Norm. Sup. Pisa 15 (1988), 615-676.

[CC3] - - - Noyaux pour résoudre l'équation $\bar{\partial}$ dans des classes ultradifférentiables sur des compacts irréguliers de $\mathbb{C}^{n}$, Proc. Mittag-Leffler Inst. 1987/1988, Math. Notes 38, Princeton Univ. Press, Princeton, 1993.

[CC4] - , Dimension de Hausdorff des ensembles de zéros et d'interpolation pour $A^{\infty}(D)$, Trans. Amer. Math. Soc. 299 (1987), 95-114.

[Ch] A.-M. Chollet, Zéros dans les classes de Gevrey de type analytique, Bull. Sci. Math. 96 (1972), 65-82.

[GrSt] P. C. Greiner and E. M. Stein, Estimates for the $\bar{\partial}$-Neumann Problem, Princeton Univ. Press, 1977.

[NSW] A. Nagel, E. M. Stein and S. Wainger, Boundary behavior of functions holomorphic in domains of finite type, Proc. Nat. Acad. Sci. U.S.A. 78 (1981), 6596-6599.

[St] E. M. Stein, Singular integrals and estimates for the Cauchy-Riemann equations, Bull. Amer. Math. Soc. 79 (1973), 440-445.

[T1] V. Thilliez, Classes de Gevrey non isotropes dans les domaines de type fini de $\mathbb{C}^{2}, \mathrm{~J}$. Analyse Math. 60 (1993), 259-305.

[T2] -, Interpolation Gevrey dans les domaines de type fini de $\mathbb{C}^{2}$, Math. Z. 212 (1993), $555-580$. 